\title{
1 Neurofunctional basis underlying audiovisual \\ 2 integration of print and speech sound in Chinese \\ 3 children
}

4 Zhichao Xia ${ }^{\text {a }} \mathrm{b}^{* \dagger}$, Ting Yang c, d* , Xin Cui a, e, Fumiko Hoeft e, f, g, h, Hong Liu c, d, f,

5 Xianglin Zhang a, Hua Shu a ${ }^{\dagger}$, Xiangping Liu c, d

7 a State Key Laboratory of Cognitive Neuroscience and Learning \& IDG/McGovern

8 Institute for Brain Research, Beijing Normal University, China

9 b School of Systems Science, Beijing Normal University, China

10 c Faculty of Psychology, Beijing Normal University, China

11 d Beijing Key Laboratory of Applied Experimental Psychology, National

12 Demonstration Center for Experimental Psychology Education, Faculty of

13 Psychology, Beijing Normal University, China

14 e Haskins Laboratories, USA

15 f Department of Psychological Sciences and Brain Imaging Research Center,

16 University of Connecticut, USA

17 g Department of Psychiatry and Weill Institute for Neurosciences and Dyslexia

18 Center, University of California, San Francisco, USA

19 h Department of Neuropsychiatry, Keio University School of Medicine, Japan

21 * These authors contributed equally to this work 
Brain basis of audiovisual integration

$23 \dagger$ Corresponding authors: Dr. Hua Shu, State Key Lab of Cognitive Neuroscience

24 and Learning, Beijing Normal University, China. Email: shuhua@bnu.edu.cn; Dr.

25 Xiangping Liu, Faculty of Psychology, Beijing Normal University, China. Email:

26 lxp599@163.com; Dr. Zhichao Xia, State Key Lab of Cognitive Neuroscience and

27 Learning, Beijing Normal University, China. Email: xiazc.psy@gmail.com

Acknowledgments: The authors would like to thank all the children and their

30 families for participating in this study and all the examiners for helping with data

31 collection. This work was supported by the Key Program of the National Social

32 Science Foundation of China (14ZDB157), National Key Basic Research Program of

33 China (2014CB846103), National Natural Science Foundation of China grants

34 (31271082, 31671126, 31611130107, 61374165, 81801782), Beijing Municipal

35 Science \& Technology Commission (Z151100003915122), Fundamental Research

36 Fund for the Central Universities, China Postdoctoral Science Foundation

37 (2019T120062, 2018M641235), and Social Science Fund of Beijing (17YYA004).

38 Conflict of Interest: The authors declare no competing financial interests.

39 Author Contributions: Conceptualization: ZX, HS, XL; Investigation: ZX, TY, XC,

40 HL, XZ; Formal Analysis: ZX; Data Curation: ZX; Writing - Original Draft

41 Preparation: ZX; Writing - Review \& Editing: ZX, TY, XC, FH, HL, XZ, HS, XL;

42 Funding Acquisition: ZX, HS, XL; Supervision: ZX, HS, XL 
bioRxiv preprint doi: https://doi.org/10.1101/2020.05.31.126128; this version posted June 17, 2021. The copyright holder for this preprint (which was not certified by peer review) is the author/funder, who has granted bioRxiv a license to display the preprint in perpetuity. It is made available under aCC-BY-NC-ND 4.0 International license.

Brain basis of audiovisual integration

43 Data Availability Statement: The data that support the findings of this study are

44 available from the corresponding authors upon reasonable request. The data are not

45 publicly available due to privacy or ethical restrictions.

46 Running Title: Brain basis of audiovisual integration 
Brain basis of audiovisual integration

\section{Abstract}

49 Effortless print-sound integration is essential to reading development, and the

50 superior temporal cortex (STC) is one most critical brain regions in alphabetic

51 languages. To examine the neural basis in a non-alphabetic language and its

52 relationship with reading ability, we conducted a functional magnetic resonance

53 imaging study in typically developing Chinese children. Audiovisual additive

54 enhancement and congruency effect in two scripts with different orthographic

55 depths_characters (opaque) and pinyin (a transparent alphabetic transcription of

56 characters)—were investigated. In terms of commonality, bilateral STCs displayed

57 additive enhancement in processing both character and pinyin stimulations.

58 Moreover, the magnitudes of congruency effect in the left STC for the two scripts

59 were highly correlated. On the other hand, differences were also observed. First,

60 areas in addition to the STC showed additive enhancement in processing pinyin-

61 sound associations. Second, while the bilateral superior temporal, left

62 inferior/middle frontal and parietal regions manifested a striking incongruency

63 effect (incongruent > congruent) for character-sound pairs, no significant clusters

64 were revealed for pinyin-sounds. Finally, the magnitude of congruency effect in the

65 left middle frontal gyrus for characters was specifically associated with silent

66 reading comprehension proficiency, indicating automatic semantic access and

67 integration during implicit character-sound processing. In contrast, the congruency

68 effect in the left STC for pinyin was specifically associated with oral reading fluency

69 that relies mainly on grapho-phonological mapping. To summarize, this study 
70 revealed both universal and script-specific neurofunctional substrates underlying

71 audiovisual integration as well as their processing- and region-dependent

72 associations with reading abilities in typical Chinese children.

\section{Keywords}

74 Audiovisual integration, character, fMRI, pinyin, Chinese, reading development

\section{$75 \quad$ Highlights}

76 - The superior temporal cortex is involved in print-sound integration regardless of

77 orthographies.

78 - The neural manifestation of audiovisual integration is modulated by

79 orthographic transparency.

80 - The congruency effect in the left middle frontal gyrus for characters is correlated

81 to reading comprehension.

82 - The congruency effect in the left superior temporal gyrus for pinyin is correlated

83 with oral reading fluency.

84 - This study provides neural evidence on the role of pinyin in Chinese reading

85 acquisition. 
Brain basis of audiovisual integration

\section{1. Introduction}

87 Efficient print-speech sound mapping is a prerequisite for and a hallmark of

88 successful reading acquisition (Blomert \& Froyen, 2010; Fabio Richlan, 2019).

89 Behavioral and neuroimaging studies have revealed that the level of automatic

90 audiovisual integration uniquely accounts for reading outcomes in both shallow

91 (e.g., Dutch, German) and opaque orthographies (e.g., English) (Bakos, Landerl,

92 Bartling, Schulte-Körne, \& Moll, 2017; Blau et al., 2010; Leo Blomert \& Willems,

93 2010; Kronschnabel, Brem, Maurer, \& Brandeis, 2014; McNorgan, Randazzo-

94 Wagner, \& Booth, 2013).

$95 \quad$ Better knowledge about the neural basis underlying audiovisual integration

96 can further our understanding of how this process contributes to reading

97 development and provide objective training targets for children with reading

98 disorders (RD). In the past two decades, functional magnetic resonance imaging

99 (fMRI) has been widely used to answer the 'where' question. Two analytic

100 approaches are the most common methods to identify brain regions involved in

101 processing multimodal information. One is based on the additive model that

102 initially examined in animal electrophysiological studies where researchers found

103 audiovisual signals induced stronger (i.e., super-additive) or lower (i.e., sub-

104 additive) neural response than the summation of responses to unimodal auditory

105 and visual inputs in areas such as the STC (Stein \& Stanford, 2008). This super-

106 additive enhancement criterion was then applied to fMRI data but proven too strict 
Brain basis of audiovisual integration

107 about detecting multisensory integration regions. In contrast, with liberal variants

108 such as mean additivity enhancement criterion (i.e., congruent condition $>$ mean of

109 unimodal auditory and visual conditions), areas including bilateral STCs can be

110 successfully identified (Beauchamp, 2005; Kronschnabel et al., 2014; McNorgan \&

111 Booth, 2015). In terms of functional significance, the super- (and mean) additive

112 enhancement occurs early and reflects general aspects of processing (Kronschnabel

113 et al., 2014; Xu, Kolozsvari, Oostenveld, Leppanen, \& Hamalainen, 2019). No

114 associations between additive enhancement with reading performance were found

115 (Kronschnabel et al., 2014). In this case, we expected that the bilateral STCs would

116 also show an additive enhancement in processing Chinese print-sound pairs.

Distinguishable brain response to congruent versus incongruent audiovisual

118 pairs is another reliable measure of multisensory integration (a.k.a. congruency

119 effect or incongruency effect if in the opposite direction) (Blau et al., 2010; Blau, van

120 Atteveldt, Ekkebus, Goebel, \& Blomert, 2009; Holloway, van Atteveldt, Blomert, \&

121 Ansari, 2015; van Atteveldt, Formisano, Goebel, \& Blomert, 2004; van Atteveldt,

122 Formisano, Goebel, \& Blomert, 2007). Compared with additive enhancement, the

123 congruency effect can reflect deep processes on the information carried by the

124 stimuli. In the pioneering study by van Atteveldt et al. (2004), the STC showed

125 stronger activation in the congruent condition compared with incongruent

126 condition. Although the involvement of STC in audiovisual integration of print and

127 speech sound has been replicated regardless of the level of processing (e.g.,

128 phoneme, syllable, or whole word) (Kronschnabel et al., 2014; McNorgan et al., 
129 2013; Wang, Karipidis, Pleisch, Fraga-Gonzalez, \& Brem, 2020), the precise

130 manifestation of the effect (e.g., direction, strength) and whether other areas

131 display such an effect are associated with multiple factors, such as orthographic

132 depth (Holloway et al., 2015; Weiyong Xu, Kolozsvari, Monto, \& Hämäläinen, 2018)

133 and reading ability (Blau et al., 2010; Blau et al., 2009; Kronschnabel et al., 2014).

134 In addition, task paradigm (McNorgan \& Booth, 2015; van Atteveldt et al., 2007),

135 stimuli properties (Kronschnabel et al., 2014), and developmental stage (Plewko et

136 al., 2018; Wang et al., 2020) also affect the congruency effect but are beyond the

137 scope of this study.

Orthographic depth describes the consistency of mapping between

139 orthographic and phonological forms (e.g., grapheme-phoneme correspondence in

140 alphabetic languages, GPC hereafter), which significantly influences behavioral and

141 neural profiles of reading development and impairments in a given writing system

142 (Borleffs, Maassen, Lyytinen, \& Zwarts, 2018; Richlan, 2014). The neural

143 processing accompanying audiovisual integration is also modulated by orthographic

144 transparency. For example, native speakers of English (deep) show an incongruency

145 effect in the STC during processing letter-sound pairs, which differs from the

146 congruency effect observed in Dutch (shallow) (Holloway et al., 2015). More

147 importantly, when the same group of English-speaking participants processed

148 numbers and number names, where the mapping was one-on-one, a strong

149 congruency effect appeared. However, the number of studies directly comparing

150 different orthographies is limited, and most existing evidence is restricted to 
151 alphabetic languages. The question of whether the same neural substrate exists in

152 non-alphabetic languages with dramatically different linguistic characteristics, such

153 as having no GPC rules, remains largely open.

Chinese is particularly suited for answering cross-linguistic questions given

155 its logographic nature and extremely deep orthography (Perfetti, Cao, \& Booth,

156 2013). Since that characters directly map to syllables and there are no GPC rules,

157 the print-sound mapping is quite arbitrary. Moreover, in many cases, different

158 characters share the same pronunciation (i.e., homophones), whereas a single

159 character may also have multiple sounds (i.e., polyphone). Therefore, the link

160 between print (character) and speech sound (syllable) is even more opaque. Based

161 on previous findings in alphabetic languages, an incongruency effect during

162 audiovisual integration was expected. In addition, grapho-semantic correspondence

163 is more systematic in Chinese characters, deeply involved in character recognition,

164 and plays a role equal to or even more important than grapho-phonological

165 processing in reading acquisition (Liu et al., 2017; Ruan, Georgiou, Song, Li, \& Shu,

166 2018; Yang, Shu, McCandliss, \& Zevin, 2013; Yang, Zevin, Shu, McCandliss, \& Li,

167 2006). Thus, it is also reasonable to expect that additional regions, such as the

168 inferior and middle frontal cortex known to be involved in semantic processing,

169 would be recruited (Wu, Ho, \& Chen, 2012; Zhao et al., 2014). Recently, Xu et al.

170 (2019) conducted a magnetoencephalography (MEG) study in Chinese adults with a

171 one-back task and identified an incongruency effect across the left frontal cortex

172 and STC. While the finding is interesting, some aspects need to be considered, 
173 including the small sample size $(\mathrm{n}=12)$, flat tone speech sounds that do not

174 correspond to any character as stimuli, and source reconstruction accuracy of MEG

175 data. Moreover, for experienced adult readers, audiovisual integration of characters

176 and corresponding sound is fully automated. Thus, in addition to cross-linguistic

177 issues, another important but unanswered question involves the neurofunctional

178 features of this process in the developing brain. Against this background, the

179 current study was to investigate the neural basis of integrating characters and

180 speech sounds in typically developing Chinese children.

182 given their fundamental role in learning-to-read in Chinese (Shu, Chen, Anderson,

$183 \mathrm{Wu}, \&$ Xuan, 2003). At the very beginning stage, a small set of pictographic

184 characters are taught first. These simple characters will later be used as radicals in

185 complex compound characters (i.e., phonograms), which comprise greater than $80 \%$

186 of Chinese characters (Chan \& Siegel, 2001). Importantly, as a pictographic

187 character does not contain any components (i.e., radicals), there is no clue on its

188 pronunciation or meaning. Thus, the mappings between its visual form with sound

189 (syllable) and meaning (morpheme) are entirely arbitrary. In addition, Mandarin

190 Chinese offers a valuable opportunity to examine the influence of orthographic

191 depth since children learn pronunciations of characters with Hanyu pinyin (pinyin

192 hereafter). In brief, pinyin is an official Roman alphabetic system used to teach the

193 sounds of characters in Mainland China and Singapore (Yin et al., 2011). A child

194 learns pinyin first once entering school and then uses it to assist in conquering the 
195 pronunciation of new characters (Guan, Liu, Chan, Ye, \& Perfetti, 2011; Shu, Peng,

196 \& McBride-Chang, 2008; Yan, Miller, Li, \& Shu, 2008). In other words, pinyin acts

197 as a scaffold during reading acquisition. Given that pinyin borrows letters from

198 alphabets and the majority have consistent mappings with speech sounds

199 (phonemes), it is pretty transparent. Therefore, we included pinyin in this study to

200 directly examine the effect of orthographic depth and expected to observe a similar

201 activation pattern in processing pinyin-sounds as in shallow languages.

In addition to investigating the neural basis of audiovisual integration at the group level, understanding how it correlates to individual heterogeneity in reading

204 abilities is important. On the one hand, children with RD showed a reduced

205 congruency effect compared with their typical peers (e.g., Blau et al., 2010; Blau et

206 al., 2009; Plewko et al., 2018). On the other hand, large individual differences in

207 reading ability are noted even within each group and can be accounted for by

208 variances at the brain level (Kast, Bezzola, Jancke, \& Meyer, 2011; McNorgan,

209 Awati, Desroches, \& Booth, 2014; McNorgan et al., 2013; Plewko et al., 2018; Wang

210 et al., 2020). Understanding such individual differences in linguistic processing,

211 which is often masked by averaging at the group level, is valuable theoretically and

212 practically (Kanai \& Rees, 2011; Kidd, Donnelly, \& Christiansen, 2018; Seghier \&

213 Price, 2018). Therefore, we also adopted an individual differences approach to

214 investigate whether neural circuitries link congruency effects in processing

215 character/pinyin-sound pairs with typical reading acquisition. 
Brain basis of audiovisual integration

\section{2. Materials and Methods}

\section{2.1. Participants}

219 The initial sample consisted of 54 typically developing children according to the

220 following inclusion criteria: (a) native speaker of Mandarin Chinese, (b) right-

221 handed, (c) normal audition, (d) normal or corrected-to-normal vision, (e) full-scale

222 intelligence quotient $(\mathrm{IQ}) \geq 80$, (f) no history of neurological or psychiatric disorders,

223 and $(\mathrm{g})$ no reading difficulties $\left(>16^{\text {th }}\right.$ percentile on the standardized reading

224 screening task Character Recognition) (Xue, Shu, Li, Li, \& Tian, 2013). All the

225 children received formal instruction on pinyin in the first half of the semester in the

$2261^{\text {st }}$ grade. Before the experiment, the children and their parents/guardians were

227 informed about the aim and procedure in detail and signed written consent. All

228 behavioral and neuroimaging data were collected during 2018. This study received

229 ethical approval from the Institutional Review Board of State Key Laboratory of

230 Cognitive Neuroscience and Learning at Beijing Normal University.

The quality of each functional run was evaluated based on in-scanner task

232 performance and the number of outliers (i.e., 'bad' data points) in blood-oxygen-

233 level-dependent signal caused by severe head motion or spikes. Acceptance

234 depended on the following criteria: (i) no more than $15 \%$ of the volumes (10 data

235 points) were marked as outliers; (ii) no less than 75\% in overall accuracy (combining

236 all the conditions) and accuracy for each condition should be no less than 50\%. The

237 run failing to meet either criterion was removed from the analysis. As a result, 36 
Brain basis of audiovisual integration

238 children had acceptable imaging data for the character experiment (23 girls; age

239 110-141 months; mean age $=127$, grades 3-5). Eighteen children were excluded due

240 to incomplete data collection $(n=9)$, poor data quality $(n=8)$, or the combination of

241 poor data quality and task performance $(n=1)$. For pinyin, the final sample

242 consisted of 41 children (27 girls; age 110-141 months; mean age = 126, grades 3-5).

243 Thirteen children were excluded due to incomplete data collection $(\mathrm{n}=6)$, poor data

244 quality $(\mathrm{n}=6)$, or the combination of poor data quality and task performance $(\mathrm{n}=$ $2451)$.

\section{$246 \quad$ 2.2. Behavior measures}

247 Each child underwent testing individually in a silent booth. IQ was measured with

248 the abbreviated version of the Chinese Wechsler Intelligence Scale for Children

249 (WISC-CR) (Wechsler, 1974). Specifically, Information, Similarities, and Digit Span

250 subtests were used to estimate verbal IQ; Picture Completion, Block Design, and

251 Coding subtests were used to estimate performance IQ.

A set of neuropsychological tasks was used to measure children's reading and

253 reading-related cognitive-linguistic skills. In brief, Character Recognition that had

254 been used for identifying Chinese dyslexic children (Cui, Xia, Su, Shu, \& Gong,

255 2016; Xia, Hoeft, Zhang, \& Shu, 2016) was used here for the screening aim. Word

256 List Reading and Silent Reading Comprehension were used to measure oral reading

257 fluency and reading comprehension proficiency, respectively. The former relies more

258 on grapho-phonological mapping, whereas the latter relies more on grapho-semantic 
Brain basis of audiovisual integration

259 processing (Xia et al., 2018). Finally, Phoneme Deletion, RAN, and Morphological

260 Production tasks were used to measure PA, RAN, and morphological awareness

261 (MA), the three critical cognitive-linguistic skills in Chinese reading development

262 (Lei et al., 2011).

264 characters children have learned. The test consisted of 150 characters selected from grades 1-6 textbooks (Xue et al., 2013). The characters were arranged in the order of

266 difficulty on ten sheets of A4 paper. During the task, the participant was asked to

267 name the characters in sequence until failing in all 15 items on one page. There was

268 no time limit. Each correct answer is worth 1 point, and the full mark is 150.

Word List Reading is a standardized test for measuring the level of oral reading fluency (Zhang et al., 2012). The test consisted of 180 high-frequency two-

271 character words arranged in a 9-column by 20-row matrix in a sheet of A4 paper.

272 The participant was required to read the words aloud in left-to-right, up-to-down

273 order as accurately and quickly as possible. The time used to complete the task was

274 recorded, and an index referring to the number of words the participant read

275 correctly per minute was calculated.

Silent Reading Comprehension is a standardized test for assessing children's

277 reading comprehension (Lei et al., 2011). The test included 100 items arranged

278 based on the number of characters (i.e., from a simple sentence to a short

279 paragraph). The participant was requested to read each sentence/paragraph silently 
Brain basis of audiovisual integration

280 and decide the correctness of the meaning with a mark of $\boldsymbol{V}$ or $\boldsymbol{X}$ as fast as possible

281 within 3 minutes. The total number of characters in sentences with correct

282 responses was calculated and then transformed to the number of characters the

283 participant read per minute.

Phoneme Deletion was used to assess PA (Li, Shu, McBride-Chang, Liu, \&

285 Peng, 2012). This task included three sections, and the participant was asked to

286 delete the initial, middle, or final sound from an orally presented syllable and

287 pronounce the remaining portion. Each section consisted of 3 practice trials and 8-

28810 testing items. One correct response is worth 1 point for a total possible score of 28928.

Digit $R A N$ required the participant to name 50 one-digit numbers on a card

291 as accurately and rapidly as possible (Liu et al., 2017). Numbers 1, 2, 3, 5, and 8

292 were repeated ten times each and arranged randomly in a 5-column by 10-row

293 matrix. The task was administered twice, and the average time the participant

294 completed the task was used as the score.

Morphological Production was used to measure MA at the character level

296 (Shu, McBride-Chang, Wu, \& Liu, 2006). In each trial, a character was orally

297 presented to the participant in a high-frequency word. The participant had to

298 respond with two new words. In one word, the given character retains the same

299 meaning as in the original case, whereas in the other word, the meaning of the 
Brain basis of audiovisual integration

300 character should be different. This task contained 15 characters for a total possible

301 score of 30.

\subsection{Stimuli and experimental design}

303 In this study, we investigated neurofunctional correlates of audiovisual integration

304 in processing character-sound and pinyin-sound pairs separately. In the character

305 experiment, the materials were characters and corresponding speech sounds

306 (syllables) (Figure 1A). Fifty-six high frequency pictographic characters were

307 selected (Chinese Single-Character Word Database;

308 https://blclab.org/pyscholinguistic-norms-database/). All the characters are visually

309 simple, taught early, and used as radicals in complex compound characters. During

310 the experiment, visual stimuli were displayed in white, 'KaiTi' font, and $96 \mathrm{pt}$ at the

311 center of a black background. A native Chinese male recorded the speech sounds

312 with a sampling rate of $44.1 \mathrm{kHz}$ and 16-bit quantization. The audio files were

313 normalized to $85 \mathrm{~dB}$ and then edited with a bandpass $(100-4000 \mathrm{~Hz})$ filter with

314 Audacity (https://www.audacityteam.org/). The average duration of all sounds was

$315476.3( \pm 87.5) \mathrm{ms}$ without pre- or post-silence. All the speech sounds can be

316 recognized easily. In the pinyin experiment, the visual stimuli were syllables

317 written in pinyin (e.g., sound /sheng 1/ can be written as 'shēng', Figure 1B), in

318 white, 'Century Schoolbook' font, and 90 pt. The auditory stimuli were the same as

319 those used in character conditions. 
Brain basis of audiovisual integration

The fMRI procedure was adapted from Blau et al. (2010) (Figure 1C). A

321 block design was used with four conditions in each experiment: unimodal auditory

322 (Aud), unimodal visual (Vis), audiovisual congruent (avC), and audiovisual

323 incongruent (avI). The visual and auditory stimuli were presented simultaneously

324 in the avC and avI conditions. The entire study contained 4 functional runs, half of

325 which were for character and the other half for pinyin. To avoid priming from

326 character processing on pinyin processing, the pinyin experiment was always

327 conducted first. Each run included 8 experimental blocks (duration 20.8 s), with

328 each condition replicated twice and interleaved with 9 rest blocks (duration $20.8 \mathrm{~s}$ ).

329 In each experimental block, there were 4 mini-blocks. Each mini-block consisted of a

$330 \quad 1.5$-s brain volume acquisition period and a 3.7-s silence period (i.e., sparse-

331 sampling) during which 4 trials of stimuli were presented. The order of the stimuli

332 and conditions were pseudorandomized. Considering the age of the participants, a

333 simple target detection task that does not require active monitoring of congruency

334 status was used to help maintain attention during scanning. Compared to the active

335 matching task, the passive paradigm will not change the automatic response

336 pattern during audiovisual integration (Blau et al., 2010; van Atteveldt et al., 2007).

337 The participant was asked to press the button with the right index finger accurately

338 and quickly to the auditory ( $440 \mathrm{~Hz}$ pure tone), visual (an unpronounceable symbol)

339 targets, or their combinations that appeared twice randomly per experimental block

340 (Figure 1D). To ensure that the child performed the task correctly, two practice 
Brain basis of audiovisual integration

341 sessions were administered, including one outside the scanner and the other before

342 the first fMRI session inside the scanner.

\subsection{Image acquisition}

344 The 3-Tesla Siemens MAGNETOM Trio Tim scanner in Imaging Center for Brain

345 Research at Beijing Normal University was used to collect all the images with a 12 -

346 channel head coil. For each child, 4 T2* sensitive gradient echo planar imaging

347 sequences were collected with the following parameters: repetition time $(\mathrm{TR})=5200$

$348 \mathrm{~ms}(1500 \mathrm{~ms}$ image acquisition plus $3700 \mathrm{~ms}$ delay), echo time $=32 \mathrm{~ms}$, flip angle $=$

34990 degrees, slice thickness $=4.5 \mathrm{~mm}$, voxel size $=3.0 \times 3.0 \times 4.5 \mathrm{~mm}^{3}$, interscan gap

$350=0.675 \mathrm{~mm}$, number of slices $=24$, number of volumes $=68$, and time of acquisition

$351=5$ minutes and $54 \mathrm{~s}$. The sparse-sampling method provided a 3.7-s period of silence

352 for stimuli presentation. Such a design is recommended for fMRI experiments

353 investigating processing with auditory inputs (Talavage \& Hall, 2012). Three

354 dummy scans were administered before image collection for each functional run to

355 avoid scanner equilibration effects. In addition, a high-resolution whole-brain T1-

356 weighted structural image (magnetization-prepared rapid acquisition with gradient

357 echo, $\mathrm{TR}=2530 \mathrm{~ms}$, echo time $=3.39 \mathrm{~ms}$, inversion time $=1100 \mathrm{~ms}$, flip angle $=7$

358 degrees, slice thickness $=1.33 \mathrm{~mm}$, voxel size $=1.33 \times 1.0 \times 1.33 \mathrm{~mm}^{3}$, number of

359 axial slices $=144$, and time of acquisition $=8$ minutes and $7 \mathrm{~s})$ was collected. Prior

360 to the formal scan, children were informed about the experimental procedure,

361 familiarized with the noise of MRI sequences, and trained to hold still. Earplugs

362 and noise-proof headphones were used to help reduce scanner noise, and foam pads 
Brain basis of audiovisual integration

363 were used to help reduce head motion during scanning. A radiologist blinded to the

364 details of the study reviewed all the images to ascertain any pathological deviations.

\section{5. fMRI preprocessing}

366 Preprocessing of brain images was conducted with SPM12 (Wellcome Department of

367 Cognitive Neurology, London, UK, http://www.fil.ion.ucl). It included the following

368 steps: (a) head motion correction (realign), (b) ART-based outlier detection (the 'bad'

369 time point was defined as intensity $>$ global mean \pm 9 SD or frame-to-frame head

370 motion [framewise displacement] $>2 \mathrm{~mm}$;

371 https://www.nitrc.org/projects/artifact_detect), (c) T1 image segmentation, (d)

372 normalization to the standard template in Montreal Neurological Institute (MNI)

373 space, and (e) smoothing with an 8-mm full-width at half-maximum Gaussian

374 kernel. No slice timing correction was performed given the discontinuous nature of

375 the signal in fMRI data collected using sparse-sampling (Perrachione \& Ghosh,

376 2013). At the individual level, experimental conditions were modeled with a

377 generalized linear model. Parameters referring to outliers in the time course and

378 head motion (3 translation, 3 rotation, and 1 framewise displacement) were

379 included in the model to exclude the effect of nuisance covariates. Then, we

380 calculated two brain maps - avC vs. (Aud + Vis) / 2 and avC vs. avI, which were

381 used in the subsequent analyses on additive enhancement and congruency effect, 382 respectively.

\subsection{Statistical analyses}


384 Descriptive statistics of demographic and behavioral measures were conducted first,

385 followed by correlations between reading abilities and cognitive-linguistic skills.

386 The Bonferroni method was used to deal with multiple comparisons (corrected $p<$

$387 \quad 0.05 / 15)$. Next, we evaluated in-scanner task performance. In this study, we

388 defined the accurate response as a button press to the target with a reaction time

389 (RT) between 200 and $2000 \mathrm{~ms}$. The average reaction time was calculated for correct

390 responses.

The two aforementioned approaches were used in the whole brain analyses to

392 identify areas involved in audiovisual processing of character and pinyin

393 stimulations separately. The first approach was to detect mean additive

394 enhancement. It consisted of two criteria: (i) stronger activation in each unimodal

395 condition against the baseline, i.e., Aud $>0$, Vis $>0$. This criterion was used to

396 exclude areas that only responded to one input modality; (ii) stronger activation in

397 the audiovisual congruent condition compared with the mean of unimodal

398 conditions, i.e., avC $>$ (Aud + Vis) / 2. For this, three one-sample $t$-tests were

399 administered, and intersections were identified. For each $t$-test, an uncorrected

400 threshold of $p$-voxel $<0.05$ was applied, resulting in a conjoint $p<0.05^{\wedge} 3=$

4010.000125 for the overlapping voxel. To be conservative, only clusters containing

402 more than 200 continuous voxels were considered to be significant. The second

403 approach was used to identify regions showing congruency effects. A one-sample $t$ -

404 test was conducted with individual contrast maps of congruency (i.e., congruent

405 against incongruent). We focused on brain areas showing activation (uncorrected $p$ - 
406 voxel < 0.05) in at least one multimodal condition to avoid results produced by

407 differences in de-activation. A threshold of $p$-voxel $<0.005$, FWE corrected $p$-cluster

$408<0.05$ was used to identify significant clusters while dealing with multiple

409 comparisons. Previous studies have repeatedly reported that the congruency effect

410 is associated with reading ability. To avoid missing the effect masked at the group

411 level, we conducted two regression analyses where the standard score of oral

412 reading fluency or silent reading comprehension proficiency was included as the

413 variate of interest, respectively. The same threshold of FWE corrected $p<0.05$

414 (cluster-forming $p$-voxel < 0.005) was used to address multiple comparisons.

415 Nuisance variables of age and sex were controlled statistically in all the analyses.

With the results of whole brain analyses, we defined areas with different

417 combinations of additive enhancement, congruency effect, and congruency-reading

418 correlation as the regions-of-interest (ROIs). Such differences may indicate different

419 roles the areas play in audiovisual integration. Given that domain-specific (i.e.,

420 reading-related) processes were of particular interest, we did not include areas that

421 only showed additive enhancement. Those regions could be more involved in

422 domain-general processing. The aims of the ROI analysis were (1) to explicitly

423 present the effects and (2) to exclude the possibility that the absence of significance

424 on a specific effect was due to the strict whole brain threshold. For this, in the

425 region only showing congruency-related effects but no additive enhancement at the

426 whole brain level, we further examined its activation in unimodal conditions and

427 the contrast avC - (Aud + Vis) / 2. In the region showing additive enhancement and 
congruency-behavior correlation but no congruency effect across the entire group at

429 the whole brain level, we examined the congruency effect with the average values

430 within the cluster. We also examined brain-behavior associations with the other

431 reading ability and cognitive-linguistic skills, while the nuisance variables of age

432 and sex were controlled statistically. Bonferroni correction was used to control the

433 number of behavior variables (corrected $p<0.05 / 4=0.0125$ ). Finally, given that

434 the left STC was identified in both the character (showing additive enhancement

435 and incongruency effect) and pinyin experiments (showing additive enhancement

436 and congruency-reading correlation), we performed conjunction analysis to examine

437 the spatial relationship between the two clusters. We also calculated the correlation

438 between the magnitudes of congruency effect in processing character-sounds and

439 processing pinyin-sounds.

441 over a FreeSurfer surface template with BrainNet Viewer (Xia, Wang, \& He, 2013).

442 The peak location was reported in MNI coordinates, and corresponding brain

443 regions were localized and reported with the AAL atlas implemented in DPABI

444 (http://rfmri.org/dpabi). Behavioral analysis and ROI analysis were performed with

445 SPSS Statistics version 24 (SPSS Inc., Chicago, IL, USA).

\section{3. Results}

\section{3.1. Behavioral profiles and in-scanner task performance}


448 Descriptive statistics of demographics, cognitive-linguistic and reading skills for all

44941 children are presented in Table 1 . Significantly positive correlations were

450 observed between reading measures (all $p$ 's $<0.05 / 15$, Bonferroni correction; see

451 Table 2 for the correlation coefficients and raw $p$-values). Regarding the

452 relationship with cognitive-linguistic skills, Word List Reading was significantly

453 associated with Digit RAN ( $r=-0.597, p<0.001<0.05 / 15)$, but not with Phoneme

454 Deletion $(r=0.288, p=0.068>0.05 / 15)$ or Morphological Production $(r=0.344, p=$

$4550.028>0.05 / 15)$ with Bonferroni correction. In contrast, Silent Reading

456 Comprehension was significantly correlated with morphological production $(r=$

$4570.487, p=0.001<0.05 / 15)$, but not with Phoneme Deletion $(r=0.135, p=0.400>$

$4580.05 / 15)$ or Digit RAN ( $r=-0.332, p=0.034>0.05 / 15)$ with Bonferroni correction.

459 This pattern indicates that in typically developing Chinese children who have

460 received formal reading instruction for 3-5 years, oral reading necessitates more

461 print-to-sound mapping, whereas reading comprehension relies more on semantic

462 processing.

Regarding the in-scanner task, all the children performed well (character

464 accuracy: $M[S D]=96.5 \%[4.8], \mathrm{RT} M[S D]=512[81] \mathrm{ms}$; pinyin accuracy $M[S D]=$

$46596.4 \%[3.7], \mathrm{RT} M[S D]=530$ [80] ms; Table S1), and no significant differences

466 were found between runs (all $p$ 's $>0.1$ ). These results indicate that participants

467 maintained attention to incoming stimuli during the entire scan session. The

468 results of ANOVA with condition as the repeated measure factor revealed no

469 significant effect on accuracy $(F=1.00, p=0.395)$ and a significant effect on RT in 
470 the character experiment $(F=35.8, p<0.001)$. Post-hoc tests revealed the longest

471 RT for the unimodal visual condition $(p$ 's $\leq 0.002$ in all comparisons, Bonferroni

472 correction), followed by the auditory condition (Aud $>$ avC: corrected $p<0.001$;

473 Aud > avI: corrected $p<0.001)$. There was no difference between the two

474 crossmodal conditions. In the pinyin experiment, the effect of condition was

475 significant on both accuracy $(F=10.1, p<0.001)$ and RT $(F=54.1, p<0.001)$. Post-

476 hoc tests revealed that while children had the lowest accuracy in the visual

477 condition ( $p$ 's $\leq 0.005$ in all comparison, Bonferroni correction), no differences were

478 found between any other pairs of conditions. In the unimodal visual condition,

479 participants also showed the longest RT (all corrected $p$ 's $\leq 0.05$ ), followed by the

480 auditory condition (Aud $>$ avC: corrected $p<0.001$; Aud $>$ avI: corrected $p<0.001$ ).

481 No differences were observed between the two crossmodal conditions.

\subsection{Brain results: the character experiment}

483 The bilateral STCs were identified as showing additive enhancement (Figure 2A;

484 Table 3; see Figure S1 for activation in two unimodal conditions and the brain

485 map for avC > (Aud + Vis) / 2). On the other hand, a significantly incongruency

486 effect (i.e., avC < avI) was found in the left frontal (peak MNI -40, 14, 32), parietal

487 (peak MNI -36, -62, 48) and bilateral STCs (peak MNI, left: -54, -22, -2; right: 62, -

488 34, 10; Figure 2B, Table 4) at the FWE corrected threshold of $p$-cluster $<0.05$. In

489 addition, whole-brain regression analysis identified a cluster in the left middle

490 frontal cortex (peak MNI -32, 10, 42) showing a positive correlation between the

491 congruency effect and silent reading comprehension proficiency at the FWE 
Brain basis of audiovisual integration

492 corrected threshold of $p$-cluster $<0.05$ (Figure 2C, Table 4). No regions were

493 identified as significant in whole-brain regression for oral reading fluency. examined their response properties and relationships with reading abilities. The

496 first type of region (bilateral STCs) showed both additive enhancement and

497 incongruency effect (Figure 2D). No congruency-reading correlations were found

498 even at uncorrected $p$-voxel $<0.05$. The second type of region (left IPL) showed only

499 incongruency effect in the whole brain analyses (Figure 2E). We found no

500 activation in either the unimodal condition or the audiovisual congruent condition.

501 No brain-behavior correlations were found in this area neither. The third type of

502 region (left MFG) showed a significant incongruency effect, of which the magnitude

503 was further associated with silent reading comprehension proficiency (Figure 2F).

504 In the ROI analysis, we found significant activation for unimodal auditory and

505 visual conditions in this area but observed no activation in the audiovisual

506 congruent condition. For the brain-behavior relationship, the magnitude of the

507 congruency also correlated with oral reading fluency $(r=0.382, p=0.026)$ at an

508 uncorrected threshold, and showed correlated trends for digit RAN $(r=-0.319, p=$

$5090.066)$ and MA $(r=0.300, p=0.084)$. Importantly, the positive correlation between

510 congruency and silent reading comprehension remained significant $(r=0.494, p=$

511 0.004) even after additionally controlling for oral reading fluency. Finally, we found

512 that the positive correlation between congruency and reading comprehension was 
513 driven by a negative correlation in the incongruent condition (avC: $r=0.084, p=$

514 0.637; avI: $r=-0.487, p=0.003)$.

\section{$515 \quad 3.3$. Brain results: the pinyin experiment}

516 The audiovisual additive enhancement was detected in the left superior temporal,

517 inferior frontal, right superior, inferior temporal, and subcortical cortices (Figure

518 3A; Table 3; see Figure $\mathbf{S 1}$ for activation in the unimodal conditions and brain

519 map for avC $>$ (Aud + Vis) / 2). No regions showed a significant congruency effect in

520 either direction in the whole brain analysis, which is unexpected. However, voxel-

521 wise regression analysis revealed that oral reading fluency was positively correlated

522 with the magnitude of congruency effect in the left STC (peak MNI -56 -36 14)

523 (FWE corrected $p$-cluster $<0.05$; Figure 3B, Table 4). No regions were identified

524 as significant in whole-brain regression for silent reading comprehension

525 proficiency. Therefore, we defined the area (left STC) showing both additive

526 enhancement and significant congruency-reading correlation as the ROI (Figure

527 3C). In the subsequent analyses, we found no congruency or incongruency effect in

528 this region even at the uncorrected threshold of $p<0.05$. In terms of brain-behavior

529 correlation, the magnitude of congruency effect was correlated with silent reading

530 comprehension without multiple comparisons correction $(r=0.371, p=0.020$,

531 uncorrected). No correlations or correlated trends were found with PA $(r=-0.087, p$

$532=0.600)$, digit RAN $(r=-0.248, p=0.127)$, or MA $(r=0.195, p=0.235)$. Of

533 importance, the correlation between the congruency effect and oral reading fluency

534 remained significant $(r=0.437, p=0.006)$ even after additionally controlling for 
535 reading comprehension. Finally, different from the left MFG observed in the

536 character experiment, the positive congruency-reading correlation in the left STC

537 was driven by a positive correlation in the congruent condition (avC: $r=0.381, p=$

$538 \quad 0.017$; avI: $r=-0.039, p=0.815)$, suggesting distinct mechanisms.

540 in both additive enhancement and congruency effect related analyses) in

541 audiovisual processing in both experiments, we conducted follow-up conjunction

542 analysis and correlation analysis to examine their relationship. First, the two ROIs

543 spatially overlapped (Figure 4A). Second, the magnitudes of the congruency effect

544 in processing character-sound pairs and pinyin-sound pairs were significantly

545 correlated $(r=0.512, p=0.002$; Figure 4B), while nuisance variables of age and sex

546 were statistically controlled.

\section{4. Discussion}

548 The present study investigated the brain bases underlying audiovisual integration

549 in characters (deep orthography) and pinyin (a transparent alphabetic coding

550 system) in typically developing Chinese children. The results revealed both script-

551 shared and script-unique neural substrates, supporting a universal principle

552 underlying the neural mechanism of reading-related processes across languages.

553 Furthermore, the evidence implies that the left MFG links audiovisual integration

554 of character-sounds with silent reading comprehension proficiency by supporting

555 automatic semantic access—reflecting a language-specific manifestation, while the 
Brain basis of audiovisual integration

556 left STG links audiovisual integration of pinyin-sounds with oral reading fluency by

557 underpinning the shared grapho-phonological mapping component—providing a

558 possible mechanism with which pinyin helps with Chinese reading development.

\subsection{Neural processes accompanying print-sound integration in scripts}

with contrasting transparencies

561 Several neuroimaging studies have investigated the neural mechanism underlying

562 print-sound integration and its role in reading development with different

563 techniques. On the one hand, electroencephalogram (EEG) and MEG were used to

564 reveal the temporal properties of neural activities (Froyen, van Atteveldt, \&

565 Blomert, 2010; Froyen, Van Atteveldt, Bonte, \& Blomert, 2008; Froyen, Bonte, van

566 Atteveldt, \& Blomert, 2009; Raij, Uutela, \& Hari, 2000; Xu et al., 2019). These

567 studies demonstrated that integration occurs preconsciously in expert reading and

568 is reflected in the component of mismatch negativity/response. On the other hand,

569 fMRI research provided more precise and detailed information about the spatial

570 localization of the processing. Among others, the bilateral STCs have been identified

571 as the most relevant areas. Like other stimuli categories, processing audiovisual

572 information of scripts induces super-additivity (or mean additive) enhancement in

573 STC (Beauchamp, 2005; McNorgan \& Booth, 2015; van Atteveldt et al., 2004).

574 Strikingly, differences in brain responses to audiovisual congruent against

575 incongruent print-sound pairs have been repeatedly reported in these areas (van

576 Atteveldt et al., 2004; van Atteveldt, Formisano, Blomert, \& Goebel, 2007). 
Brain basis of audiovisual integration

577 Importantly, such effects were observed in passive paradigms, pinpointing the

578 automatic nature of this effect (Blau et al., 2010; van Atteveldt et al., 2007).

Additive enhancement is more domain-general and has been reported in the

580 left STC in both transparent (van Atteveldt et al., 2004) and opaque languages

581 (McNorgan \& Booth, 2015). Consistent with previous studies, we observed

582 audiovisual additive enhancement in the bilateral STCs in processing both

583 character-sound (deep) and pinyin-sound (shallow) associations. Compared with

584 additive enhancement, the congruency effect reflects more domain-specific

585 processing and is affected by factors, such as linguistic characteristics (Holloway et

586 al., 2015) and reading abilities (Blau et al., 2010; Blau et al., 2009; Karipidis et al.,

587 2018). Consistency of grapheme-phoneme mapping (orthographic depth or

588 transparency) is an essential feature in alphabetic languages. Differences in the

589 direction of the congruency effect and brain regions showing this effect have been

590 reported between languages with different orthographic depths (Blomert \& Froyen,

591 2010; Holloway et al., 2015). In particular, greater activation in the congruent

592 condition than incongruent condition is more consistently reported in skilled

593 readers when processing letter-sound associations in transparent scripts. In

594 contrast, the opposite pattern is observed in opaque scripts (Holloway et al., 2015).

595 Chinese is a logographic language with an extremely deep orthography. Therefore,

596 we expected to observe an incongruency effect. In support of this hypothesis, the

597 results of the character experiment revealed that four regions (including the

598 bilateral STC) displayed significant incongruency effects. In contrast, in the absence 
599 of a congruency or incongruency effect in processing pinyin-sound pairs at the group

600 level, we observed a positive correlation between the magnitude of congruency effect

601 and oral reading fluency. Higher skill readers showed a congruency effect, whereas

602 lower skill readers showed an incongruency effect. This pattern is consistent with

603 previous studies that revealed the lack of a congruency effect in dyslexia in

604 transparent languages (Blau et al., 2010; Blau et al., 2009), as well as these

605 investigated brain-behavior correlations at both the phoneme (Karipidis et al., 2016;

606 Karipidis et al., 2018) and syllable levels (McNorgan et al., 2014; Wang et al., 2020).

607 4.2. Left frontal and parietal cortex in implicitly integrating audiovisual 608 information of Chinese characters

609 Opaque orthography is not the only linguistic feature that affects the neural

610 processing of character-sound processing. Chinese characters are morpheme-

611 syllabic, which means that semantic information is deeply involved. Therefore, in

612 addition to grapho-phonological mapping, semantic access is an equally or even

613 more important aspect in reading (Bi, Han, Weekes, \& Shu, 2007; Dang, Zhang,

614 Wang, \& Yang, 2018; Liu et al., 2017; Ruan et al., 2018; Yang et al., 2013; Yang et

615 al., 2006). In this study, the left MFG displayed a significant incongruency effect

616 and showed a unique association between the magnitude of the effect and silent

617 reading comprehension proficiency. Considering that this region did not show

618 additive enhancement, we speculate that the observed incongruency effect is more

619 likely to be downstream and reflects semantic processing. In previous research,

620 frontal areas have also been reported along with a broad bilateral fronto-parietal 
621 network. Its function was proposed as task-related top-down modulation (van

622 Atteveldt et al., 2007) or implicit domain-general conflict detection and resolution

623 (Holloway et al., 2015). Recently, the incongruency effect measured with MEG was

624 sourced to the frontal cortex in experienced Chinese adult readers (Xu et al., 2019).

625 Here, we replicated this finding by revealing the incongruency effect in the left

626 MFG in a developmental population in Chinese with fMRI and further

627 demonstrated that the role of this region in the implicit audiovisual integration of

628 characters is domain-specific and that the information automatically processed in

629 this region corresponds to semantics. This finding is also consistent with the opinion

630 that while the fundamental neural circuit for reading is universal, linguistic

631 features in a given language introduce different demands for specific cognitive

632 components (Perfetti et al., 2013; Rueckl et al., 2015).

633 In Chinese, neither grapho-semantic mapping nor phono-semantic mapping

634 is reliable at the character level. Given that no activation was found in the

635 audiovisual congruent condition for characters, the incongruency effect in the left

636 frontal cortex is more likely to reflect a resolution of the conflict between different

637 meanings accessed via visual and auditory inputs. Interestingly, the positive

638 correlation between the magnitude of congruency effect and reading comprehension

639 proficiency was driven by a negative correlation in the incongruent condition. In

640 other words, individuals with better comprehension performance showed lower

641 activation for non-matching print-sound pairs. This pattern could be caused by

642 higher neural efficiency in relevant processing, such as implicit suppression of 
643 conflicting semantic information. It has previously been found that in the initial

644 stage of establishing a binding between grapheme and phoneme in Swiss-German,

645 typical readers show higher activation in incongruent conditions, and such

646 activation decreases as automation is achieved (Plewko et al., 2018).

647 In addition to the bilateral temporal and left frontal cortices, a left parietal

648 area also displayed a significant incongruency effect. Like the frontal region, this

649 region did not show additive enhancement, implying that higher activation in the

650 incongruent condition is more likely to be a downstream effect. Unlike the left MFG,

651 no activation was observed in either the unimodal auditory or visual condition, and

652 there was no congruency-reading correlation. Therefore, although previous studies

653 regarded the left parietal cortex as a part of the semantic network (Binder \& Desai,

654 2011; Wang, Zhao, Zevin, \& Yang, 2016), there was an absence of evidence

655 indicating that the incongruency effect in this area reflects semantic processing. In

656 another line of research, compared with English, the left parietal region was more

657 recruited in Chinese reading for visual orthographic analysis given the visual

658 complexity of characters (Perfetti et al., 2013). In this case, the incongruency effect

659 might reflect a conflict of orthographic information. However, here we found no

660 activation in the visual condition, going against this explanation. Third, although

661 the left partial cortex has not been associated with print-sound integration

662 previously in one's native language, a recent training study revealed that this

663 region was involved in acquiring novel grapho-phonological mapping (Xu, 
Brain basis of audiovisual integration

664 Kolozsvari, Oostenveld, \& Hamalainen, 2020). As its function in audiovisual

665 processing remains unclear, future studies are needed.

666 4.3. The left STG links implicit integration of pinyin-sound pairs with oral

667 reading fluency

668 While conquering the sounds of letters to an automatic level is one of the most

669 critical prerequisites in alphabetic languages (Blomert, 2011; Richlan, 2019),

670 learning pinyin—a transparent phonological coding system—plays a scaffolding role

671 at the initial stage of Chinese reading acquisition in Mainland China. Therefore, the

672 present study not only examined whether and how the neural features of

673 audiovisual integration are associated with orthographic transparency but also

674 provided information that helps illustrate the role of pinyin in developing fluent

675 reading ability in Chinese.

$676 \quad$ First, widespread brain regions, including the bilateral STCs, showed

677 additive enhancement in processing pinyin-sound associations, in line with previous

678 findings in alphabetic languages (Kronschnabel et al., 2014; McNorgan \& Booth,

679 2015). For the congruency effect, we expected to observe a stronger activation in the

680 congruent condition than in the incongruent condition in the STC based on previous

681 studies in transparent scripts (Blau et al., 2010; van Atteveldt et al., 2004). At first

682 glance, no significant congruency or incongruency effect was observed. There could

683 be several reasons. First, the stimuli we used were at the syllable level. Differences

684 between the letter-phoneme integration and string-syllable integration have been 
685 reported in previous studies (Kronschnabel et al., 2014). However, the authors also

686 found that neural response to word-like stimuli is more sensitive to reading

687 abilities. Thus, the alternative possibility is that the direction and strength of the

688 congruency effect are associated with reading abilities and masked by group

689 averaging. In line with the latter, we did observe a positive correlation between the

690 magnitude of the congruency effect in the left STG and oral reading fluency.

691 Specifically, children with higher oral reading scores are more likely to show

692 stronger congruency effects. In contrast, those with lower scores are more likely to

693 show weaker congruency effects or even incongruency effects. This finding is

694 consistent with studies, where a reduced congruency effect was found in individuals

695 with RD in shallow languages (Blau et al., 2010; Blau et al., 2009), studies exploring

696 brain-behavior correlation in transparent (Wang et al., 2020), and opaque languages

697 (McNorgan et al., 2014), as well as studies using a training paradigm (Karipidis et

698 al., 2016; Karipidis et al., 2018). The left STG is the central area representing

699 phonological information (Boets et al., 2013; Glezer et al., 2016) and is involved in

700 visual phonological processing in Chinese children and adults (Cao et al., 2010). Its

701 activation and connectivity change after learning visual-sound mappings (Dehaene

702 et al., 2010; Li, Xu, Luo, Zeng, \& Han, 2020; Thiebaut de Schotten, Cohen,

703 Amemiya, Braga, \& Dehaene, 2014), which can further scaffold later reading

704 development (Wang, Joanisse, \& Booth, 2020). Noteworthily, the positive

705 correlation between the congruency effect in the left STG for pinyin-sound pairs and

706 oral reading fluency was driven by a positive correlation in the congruent condition. 
Brain basis of audiovisual integration

707 This pattern is different from that in the left MFG in processing character-sound

708 pairs, indicating different mechanisms. It should also be noted that the current

709 findings were revealed with typical readers. Whether children with RD have deficits

710 or show other processing patterns requires further investigation.

711 The significant correlation between the magnitude of congruency effect in the

712 left STG to pinyin-sound pairs with oral reading fluency implies that learning

713 pinyin helps children develop the related cognitive components and underlying

714 neural circuits for fluent oral reading (though causal claims cannot be made from

715 this study). Compared with cognitive-linguistic skills such as PA and RAN, less

716 attention has been given to pinyin processing in understanding Chinese reading

717 development, probably because of its auxiliary role as a scaffold in reading

718 acquisition but not reading itself. However, although the number of studies is

719 limited, the pinyin-reading relationship has been demonstrated (Lin et al., 2010;

720 Pan et al., 2011; Siok \& Fletcher, 2001; Zhang, Georgiou, Inoue, Zhong, \& Shu,

721 2020). Pan et al. found that invented pinyin spelling at age 6 independently

722 predicted reading performance at ages 8 and 10 (Pan et al., 2011). Zhang and

723 colleagues' recent finding suggests a bidirectional relationship (Zhang et al., 2020).

724 Several explanations were proposed based on behavioral evidence. One is that

725 pinyin can help to establish better phonological awareness, the cognitive processing

726 shared by pinyin and character reading (Ding, Liu, McBride, \& Zhang, 2015; Li et

727 al., 2020; McBride, Wang, \& Cheang, 2018; Shu et al., 2008; Yin et al., 2011). Above

728 and beyond PA, there is also a unique contribution from pinyin processing skills to 
729 character reading (Ju, Zhou, \& delMas, 2021). It is reasonable to hypothesize that

730 pinyin may also help establish the correspondence between print and sound. In

731 support of this notion, a study revealed that training on pinyin uniquely improved

732 character-to-syllable mapping in Chinese as a second language learner (Guan et al.,

733 2011). In the present study, a spatially overlapping region also showed additive

734 enhancement and incongruency effect in processing characters. Furthermore, we

735 found that the magnitudes of congruency effect in processing two types of stimuli

736 were highly correlated. As the left posterior STC is involved in visual rhyming

737 judgment of Chinese characters and proposed to play a role in mapping between

738 orthography and phonology (Cao et al., 2010), these findings collectively suggest

739 that learning pinyin may shape the neural circuit for print-sound integration that

740 will later be recruited in learning characters.

\section{4.4. Caveats and future directions}

742 While this study investigated the neurofunctional basis of integrating audiovisual

743 information of characters and pinyin, representing one of the first efforts, caution

744 should be considered when interpreting the results. First, we focused on print-sound

745 integration at the syllable level, whereas previous studies mainly explored letter-

746 speech sound associations (but see Kronschnabel et al., 2014; Wang et al., 2020).

747 This is because Chinese has no GPC rules, and print-sound mapping occurs at the

748 syllable level. But similar to GPC rules in alphabetic languages, simple characters

749 are taught at the earliest stages and play fundamental roles in reading

750 development (Shu et al., 2003; Xing, Shu, \& Li, 2004). Second, because our 
751 participants were in grades $3-5$, pinyin may play a different role than it does in

752 earlier stages of reading development. Siok and Fletcher (2001) unveiled significant

753 correlations between pinyin knowledge and reading in grades 2-5, but not in grade

754 1. Thus, it could be the particular age range that enabled us to observe the

755 relationship between print-sound processing in pinyin and oral reading fluency. To

756 further address this issue, it is necessary to conduct studies following preliterate

757 children until they achieve fluent reading. Finally, in this study, we uncovered the

758 brain bases of print-sound integrations in two contrasting scripts and their

759 relationship with reading performance in typically developing children. One next

760 question that warrants investigation in the future is whether neural processes are

761 altered in children with RD.

\section{5. Conclusion}

763 In the current study, we investigated regions that are involved in audiovisual

764 integration of character-sounds and pinyin-sounds. The results suggest the bilateral

765 STCs as the universal brain substrates underlying audiovisual integration across

766 scripts. At the same time, the precise manifestation of specific effects is influenced

767 by linguistic features in the given language. In particular, the left MFG is strongly

768 involved in the audiovisual integration of characters and correlated with reading

769 comprehension, suggesting that semantic representations were more efficiently

770 accessed and processed in readers with higher comprehension proficiency. In

771 contrast, the brain-behavior correlation in the left STC implies that pinyin may 
Brain basis of audiovisual integration

772 help children develop better reading skills by shaping the shared component of

773 grapho-phonological mapping and shaping the underlying brain circuits.

\section{References}

775 Bakos, S., Landerl, K., Bartling, J., Schulte-Körne, G., \& Moll, K. (2017). Deficits in Dyslexia: Results from a Novel ERP-Paradigm. Front Hum Neurosci, 11(116).

Beauchamp, M. S. (2005). Statistical criteria in FMRI studies of multisensory integration. Neuroinformatics, 3(2), 93-113.

Bi, Y., Han, Z., Weekes, B., \& Shu, H. (2007). The interaction between semantic and the nonsemantic systems in reading: Evidence from Chinese. Neuropsychologia, 45(12), 2660-2673.

783 Binder, J. R., \& Desai, R. H. (2011). The neurobiology of semantic memory. Trends Cogn Sci, 15(11), 527-536.

Blau, V., Reithler, J., van Atteveldt, N., Seitz, J., Gerretsen, P., Goebel, R., \& Blomert, L. (2010). Deviant processing of letters and speech sounds as proximate cause of reading failure: a functional magnetic resonance imaging

789 Blau, V., van Atteveldt, N., Ekkebus, M., Goebel, R., \& Blomert, L. (2009). Reduced neural integration of letters and speech sounds links phonological and 
Brain basis of audiovisual integration

Blomert, L. (2011). The neural signature of orthographic-phonological binding in successful and failing reading development. Neuroimage, 57(3), 695-703.

794 Blomert, L., \& Froyen, D. (2010). Multi-sensory learning and learning to read. Int $J$ Psychophysiol, 77(3), 195-204.

796 Blomert, L., \& Willems, G. (2010). Is there a causal link from a phonological awareness deficit to reading failure in children at familial risk for dyslexia? Dyslexia, 16(4), 300-317.

799 Boets, B., Op de Beeck, H. P., Vandermosten, M., Scott, S. K., Gillebert, C. R., 800 Mantini, D., . . Ghesquiere, P. (2013). Intact but less accessible phonetic 801 representations in adults with dyslexia. Science, 342(6163), 1251-1254.

802 Borleffs, E., Maassen, B. A. M., Lyytinen, H., \& Zwarts, F. (2018). Cracking the Code: The Impact of Orthographic Transparency and Morphological-Syllabic Complexity on Reading and Developmental Dyslexia. Front Psychol, 9(2534), 2534.

806 Cao, F., Lee, R., Shu, H., Yang, Y. H., Xu, G. Q., Li, K. C., \& Booth, J. R. (2010).

807 Cultural Constraints on Brain Development: Evidence from a Developmental 808 Study of Visual Word Processing in Mandarin Chinese. Cereb Cortex, 20(5), 1223-1233.

810 Chan, C. K. K., \& Siegel, L. S. (2001). Phonological Processing in Reading Chinese 811 among Normally Achieving and Poor Readers. J Exp Child Psychol, 80(1), 23- 
Brain basis of audiovisual integration

813 Cui, Z., Xia, Z., Su, M., Shu, H., \& Gong, G. (2016). Disrupted white matter connectivity underlying developmental dyslexia: A machine learning approach. Human Brain Mapping, 37(4), 1443-1458.

816 Dang, M., Zhang, R., Wang, X., \& Yang, J. (2018). The Interaction Between Psychol, 9, 2748.

Dehaene, S., Pegado, F., Braga, L. W., Ventura, P., Nunes, G., Jobert, A., . . Cohen, and Language. Science, 330(6009), 1359-1364.

Ding, Y., Liu, R. D., McBride, C., \& Zhang, D. (2015). Pinyin Invented Spelling in Mandarin Chinese-Speaking Children With and Without Reading Difficulties. J Learn Disabil, 48(6), 635-645.

825 Froyen, D., van Atteveldt, N., \& Blomert, L. (2010). Exploring the Role of Low Level Visual Processing in Letter-Speech Sound Integration: A Visual MMN Study.

828 Froyen, D., Van Atteveldt, N., Bonte, M., \& Blomert, L. (2008). Cross-modal enhancement of the MMN to speech-sounds indicates early and automatic

831 Froyen, D. J. W., Bonte, M. L., van Atteveldt, N., \& Blomert, L. (2009). The Long 832 Road to Automation: Neurocognitive Development of Letter-Speech Sound 833 Processing. J Cogn Neurosci, 21(3), 567-580. 
834 Glezer, L. S., Eden, G., Jiang, X., Luetje, M., Napoliello, E., Kim, J., \& Riesenhuber, M. (2016). Uncovering phonological and orthographic selectivity across the reading network using fMRI-RA. Neuroimage, 138, 248-256.

837 Guan, C. Q., Liu, Y., Chan, D. H. L., Ye, F., \& Perfetti, C. A. (2011). Writing strengthens orthography and alphabetic-coding strengthens phonology in learning to read Chinese. Journal of Educational Psychology, 103(3), 509-522. Dependency in the Neural Correlates of Reading: Evidence from Audiovisual Integration in English Readers. Cereb Cortex, 25(6), 1544-1553.

843 I Karipidis, I., Pleisch, G., Röthlisberger, M., Hofstetter, C., Dornbierer, D.,

844 Stämpfli, P., \& Brem, S. (2016). Neural initialization of audiovisual integration in prereaders at varying risk for developmental dyslexia. Human Brain Mapping.

847 Ju, Z., Zhou, Y., \& delMas, R. (2021). The contributions of separate pinyin skills and oral vocabulary to Chinese word reading of U.S. Mandarin immersion third graders. Reading and Writing.

850 Kanai, R., \& Rees, G. (2011). The structural basis of inter-individual differences in human behaviour and cognition. Nature Reviews Neuroscience, 12(4), 231242.

853 Karipidis, I. I., Pleisch, G., Brandeis, D., Roth, A., Röthlisberger, M., Schneebeli, M., 
Brain basis of audiovisual integration

reading outcome and multimodal brain signatures of letter-speech sound learning in prereaders. Scientific Reports, 8(1), 7121.

857 Kast, M., Bezzola, L., Jancke, L., \& Meyer, M. (2011). Multi- and unisensory dyslexic and nondyslexic adults. Brain and Language, 119(3), 136-148.

860 Kidd, E., Donnelly, S., \& Christiansen, M. H. (2018). Individual Differences in Language Acquisition and Processing. Trends Cogn Sci, 22(2), 154-169.

862 Kronschnabel, J., Brem, S., Maurer, U., \& Brandeis, D. (2014). The level of audiovisual print-speech integration deficits in dyslexia. Neuropsychologia,

865 Lei, L., Pan, J., Liu, H., McBride-Chang, C., Li, H., Zhang, Y., . . Shu, H. (2011).

866 Developmental trajectories of reading development and impairment from ages 3 to 8 years in Chinese children. J Child Psychol Psychiatry, 52(2), 212220. morphological skills. Journal of Research in Reading, 35(3), 287-307. for novel stimuli selectively activates the neural network of the visual word form area. Neuroimage, 215, 116838. 
Brain basis of audiovisual integration

Effects on Pinyin skills, phonological awareness, and character reading. Dyslexia, 26(4), 377-393.

879 Lin, D., McBride-Chang, C., Shu, H., Zhang, Y., Li, H., Zhang, J., . . Levin, I.

882 Liu, Y., Georgiou, G. K., Zhang, Y., Li, H., Liu, H., Song, S., . . Shu, H. (2017). fluency in Chinese. International Journal of Educational Research, 82, 75-90.

885 McBride, C., Wang, Y., \& Cheang, L. M.-L. (2018). Dyslexia in Chinese. Current Developmental Disorders Reports, 5(4), 217-225.

887 McNorgan, C., Awati, N., Desroches, A. S., \& Booth, J. R. (2014). Multimodal

890 McNorgan, C., \& Booth, J. R. (2015). Skill dependent audiovisual integration in the fusiform induces repetition suppression. Brain and Language, 141, 110-123.

892 McNorgan, C., Randazzo-Wagner, M., \& Booth, J. R. (2013). Cross-modal integration in the brain is related to phonological awareness only in typical readers, not in those with reading difficulty. Front Hum Neurosci, 7(388),

896 Pan, J., McBride-Chang, C., Shu, H., Liu, H., Zhang, Y., \& Li, H. (2011). What is in the naming? A 5-year longitudinal study of early rapid naming and 
Brain basis of audiovisual integration Psychology, 103(4), 897-908.

901 Perfetti, C., Cao, F., \& Booth, J. (2013). Specialization and Universals in the

904 Perrachione, T. K., \& Ghosh, S. S. (2013). Optimized Design and Analysis of Sparse-

906 Plewko, J., Chyl, K., Bola, Ł., Łuniewska, M., Dębska, A., Banaszkiewicz, A., . . .

909 Raij, T., Uutela, K., \& Hari, R. (2000). Audiovisual integration of letters in the human brain. Neuron, 28(2), 617-625.

911 Richlan, F. (2014). Functional neuroanatomy of developmental dyslexia: the role of orthographic depth. Front Hum Neurosci, 8, 347.

913 Richlan, F. (2019). The Functional Neuroanatomy of Letter-Speech Sound

914 Integration and Its Relation to Brain Abnormalities in Developmental

915 Dyslexia. Front Hum Neurosci, 13(21).

916 Ruan, Y., Georgiou, G. K., Song, S., Li, Y., \& Shu, H. (2018). Does writing system

917 influence the associations between phonological awareness, morphological

918 awareness, and reading? A meta-analysis. Journal of Educational

$919 \quad$ Psychology, 110(2), 180. 
Brain basis of audiovisual integration

920 Rueckl, J. G., Paz-Alonso, P. M., Molfese, P. J., Kuo, W. J., Bick, A., Frost, S. J., . . Frost, R. (2015). Universal brain signature of proficient reading: Evidence from four contrasting languages. Proc Natl Acad Sci U S A, 112(50), 15510-

923 15515.

924 Seghier, M. L., \& Price, C. J. (2018). Interpreting and Utilising Intersubject Variability in Brain Function. Trends Cogn Sci, 22(6), 517-530.

928 Shu, H., McBride-Chang, C., Wu, S., \& Liu, H. Y. (2006). Understanding Chinese developmental dyslexia: Morphological awareness as a core cognitive construct. Journal of Educational Psychology, 98(1), 122-133.

931 Shu, H., Peng, H., \& McBride-Chang, C. (2008). Phonological awareness in young Chinese children. Dev Sci, 11(1), 171-181.

933 Siok, W. T., \& Fletcher, P. (2001). The role of phonological awareness and visualorthographic skills in Chinese reading acquisition. Dev Psychol, 37(6), 886899.

936 Stein, B. E., \& Stanford, T. R. (2008). Multisensory integration: current issues from the perspective of the single neuron. Nat Rev Neurosci, 9(4), 255-266.

938 Talavage, T. M., \& Hall, D. A. (2012). How challenges in auditory fMRI led to general advancements for the field. Neuroimage, 62(2), 641-647. 
Brain basis of audiovisual integration

940 Thiebaut de Schotten, M., Cohen, L., Amemiya, E., Braga, L. W., \& Dehaene, S.

941 (2014). Learning to read improves the structure of the arcuate fasciculus.

$942 \quad$ Cereb Cortex, 24(4), 989-995.

943 van Atteveldt, N., Formisano, E., Goebel, R., \& Blomert, L. (2004). Integration of

$944 \quad$ letters and speech sounds in the human brain. Neuron, 43(2), 271-282.

945 van Atteveldt, N. M., Formisano, E., Blomert, L., \& Goebel, R. (2007). The effect of

946 temporal asynchrony on the multisensory integration of letters and speech

$947 \quad$ sounds. Cereb Cortex, 17(4), 962-974.

948 van Atteveldt, N. M., Formisano, E., Goebel, R., \& Blomert, L. (2007). Top-down

949 task effects overrule automatic multisensory responses to letter-sound pairs

950 in auditory association cortex. Neuroimage, 36(4), 1345-1360.

951 Wang, F., Karipidis, II, Pleisch, G., Fraga-Gonzalez, G., \& Brem, S. (2020).

952 Development of Print-Speech Integration in the Brain of Beginning Readers

$953 \quad$ With Varying Reading Skills. Front Hum Neurosci, 14(289), 289.

954 Wang, J., Joanisse, M. F., \& Booth, J. R. (2020). Neural representations of

955 phonology in temporal cortex scaffold longitudinal reading gains in 5- to 7-

$956 \quad$ year-old children. Neuroimage, 207, 116359.

957 Wang, X., Zhao, R., Zevin, J. D., \& Yang, J. (2016). The Neural Correlates of the

958 Interaction between Semantic and Phonological Processing for Chinese

$959 \quad$ Character Reading. Front Psychol, 7.

960 Wechsler, D. (1974). WISC-R, Wechsler intelligence scale for children, revised:

$961 \quad$ Psychological Corporation. 
Brain basis of audiovisual integration

962 Wu, C. Y., Ho, M. H., \& Chen, S. H. (2012). A meta-analysis of fMRI studies on 63(1), 381-391.

Xia, M., Wang, J., \& He, Y. (2013). BrainNet Viewer: a network visualization tool for human brain connectomics. PLoS One, 8(7), e68910.

967 Xia, Z., Hoeft, F., Zhang, L., \& Shu, H. (2016). Neuroanatomical anomalies of dyslexia: Disambiguating the effects of disorder, performance, and maturation. Neuropsychologia, 81, 68-78.

970 Xia, Z., Zhang, L., Hoeft, F., Gu, B., Gong, G., \& Shu, H. (2018). Neural correlates of oral word reading, silent reading comprehension, and cognitive subcomponents. International Journal of Behavioral Development, O(0), 0165025417727872 .

974 Xing, H., Shu, H., \& Li, P. (2004). The acquisition of Chinese characters: Corpus analyses and connectionist simulations. Journal of Cognitive Science, 5(1), 1-

977 Xu, W., Kolozsvari, O. B., Monto, S. P., \& Hämäläinen, J. A. (2018). Brain Responses to Letters and Speech Sounds and Their Correlations With Cognitive Skills Related to Reading in Children. Front Hum Neurosci,

981 Xu, W., Kolozsvari, O. B., Oostenveld, R., \& Hamalainen, J. A. (2020). Rapid 982 changes in brain activity during learning of grapheme-phoneme associations $983 \quad$ in adults. Neuroimage, 220, 117058. 
Brain basis of audiovisual integration

984 Xu, W., Kolozsvari, O. B., Oostenveld, R., Leppanen, P. H. T., \& Hamalainen, J. A. (2019). Audiovisual Processing of Chinese Characters Elicits Suppression and Congruency Effects in MEG. Front Hum Neurosci, 13(18), 18.

987 Xue, J., Shu, H., Li, H., Li, W., \& Tian, X. J. J. o. P. R. (2013). The Stability of Literacy-Related Cognitive Contributions to Chinese Character Naming and Reading Fluency. 42(5), 433-450.

Yan, M., Miller, K. F., Li, H., \& Shu, H. (2008). What is the place for Pinyin in beginning Chinese reading? Evidence from eye movements. In Cognitive and cultural influences on eye movements. (pp. 343-360). Tianjin, China: Tianjin

994 Yang, J., Shu, H., McCandliss, B. D., \& Zevin, J. D. (2013). Orthographic influences on division of labor in learning to read Chinese and English: Insights from computational modeling. Biling (Camb Engl), 16(Spec Iss 2), 354-366.

997 Yang, J., Zevin, J. D., Shu, H., McCandliss, B. D., \& Li, P. (2006). A "triangle model" of Chinese reading. Paper presented at the 28 th annual conference of the

1000 Yin, L., Li, W., Chen, X., Anderson, R. C., Zhang, J., Shu, H., \& Jiang, W. (2011). The role of tone awareness and pinyin knowledge in Chinese reading. Writing Systems Research, 3(1), 59-68.

1003 Zhang, S., Georgiou, G. K., Inoue, T., Zhong, W., \& Shu, H. (2020). Do pinyin and 
bioRxiv preprint doi: https://doi org/10.1101/2020 05.31.126128; this version posted June 17, 2021. The copyright holder for this preprint (which was not certified by peer review) is the author/funder, who has granted bioRxiv a license to display the preprint in perpetuity. It is made available under aCC-BY-NC-ND 4.0 International license.

Brain basis of audiovisual integration

1006 Zhang, Y., Zhang, L., Shu, H., Xi, J., Wu, H., Zhang, Y., \& Li, P. (2012). Universality

1007 of categorical perception deficit in developmental dyslexia: an investigation of

1008 Mandarin Chinese tones. Journal of Child Psychology and Psychiatry, 53(8),

$1009 \quad 874-882$

1010 Zhao, J., Wang, X., Frost, S. J., Sun, W., Fang, S.-Y., Mencl, W. E., . . Rueckl, J. G.

1011 (2014). Neural division of labor in reading is constrained by culture:

1012 A training study of reading Chinese characters. Cortex, 53, 90-106.

1013 
Table 1 Demographics and behavior profiles $(n=41)$

\begin{tabular}{|c|c|c|c|}
\hline Measure & Mean & $S D$ & Range \\
\hline Age (month) & 126 & 8 & $110-141$ \\
\hline Grade $\left(3^{\text {rd }} / 4^{\text {th }} / 5^{\text {th }}\right)$ & & $4 / 23 / 14$ & \\
\hline Sex (female / male) & & $27 / 14$ & \\
\hline Verbal IQ (standard score) & 107 & 13 & $85-145$ \\
\hline Performance IQ (standard score) & 114 & 13 & $75-137$ \\
\hline Full-scale IQ (standard score) & 111 & 12 & $80-143$ \\
\hline Character Recognition (corrected item) & 119 & 12 & $86-146$ \\
\hline Word List Reading (word / minute) & 90 & 19 & $55-140$ \\
\hline Silent Reading Comprehension (character / & 327 & 119 & $132-565$ \\
\hline \multicolumn{4}{|l|}{ minute) } \\
\hline Phoneme Deletion (corrected item) & 21 & 4 & $7-25$ \\
\hline Rapid Naming (second) & 18 & 3 & $11-24$ \\
\hline Morphological Production (item) & 24 & 4 & $13-30$ \\
\hline \multicolumn{4}{|l|}{ Number of 'bad' time points per fMRI run } \\
\hline Character Experiment a & 2.4 & 3.37 & $0-10$ \\
\hline Pinyin Experiment & 0.74 & 1.54 & $0-9$ \\
\hline
\end{tabular}

Note. ${ }^{\text {a }} \mathrm{n}=36$ 
Table 2 Correlation between reading and cognitive-linguistic skills $(\mathrm{n}=41)$

\begin{tabular}{|c|c|c|c|c|c|c|}
\hline Measures & 1 & 2 & 3 & 4 & 5 & 6 \\
\hline 1 Character Recognition & $+\cdots$ & & & & & \\
\hline \multirow[t]{2}{*}{2 Word List Reading } & $r=0.559$ & -- & & & & \\
\hline & $p<0.001$ & & & & & \\
\hline \multirow[t]{2}{*}{3 Silent Reading Comprehension } & $r=0.647$ & $r=0.701$ & -- & & & \\
\hline & $p<0.001$ & $p<0.001$ & & & & \\
\hline \multirow[t]{2}{*}{4 Phoneme Deletion } & $r=0.286$ & $r=0.288$ & $r=0.135$ & -- & & \\
\hline & $p=0.070$ & $p=0.068$ & $p=0.400$ & & & \\
\hline \multirow[t]{2}{*}{5 Rapid Naming } & $r=-0.357$ & $r=-0.597$ & $r=-0.332$ & $r=-0.160$ & $\cdots$ & \\
\hline & $p=0.022$ & $p<0.001$ & $p=0.034$ & $p=0.319$ & & \\
\hline \multirow[t]{2}{*}{6 Morphological Production } & $r=0.566$ & $r=0.344$ & $r=0.487$ & $r=0.122$ & $r=-0.117$ & $\cdots$ \\
\hline & $p<0.001$ & $p=0.028$ & $p=0.001$ & $p=0.446$ & $p=0.466$ & \\
\hline
\end{tabular}


Table 3 Cluster identified as showing audiovisual additive enhancement in each experiment

\begin{tabular}{lll}
\hline Experiment & \# Voxel & Brain area \\
\hline Character & 822 & Temporal_Sup_R, Temporal_Mid_R \\
& 354 & Temporal_Sup_L, Temporal_Mid_L \\
& & Temporal_Mid_L, Temporal_Sup_L, Rolandic_Oper_L, Occipital_Mid_L, Temporal_Inf_L, \\
Pinyin & 2752 & SupraMarginal_L, Fusiform_L, Postcentral_L, Heschl_L, Temporal_Pole_Sup_L, Occipital_Inf_L \\
& & Temporal_Sup_R, Temporal_Mid_R, SupraMarginal_R \\
& 967 & Temporal_Mid_R, Temporal_Inf_R, Fusiform_R, Cerebelum_6_R, Occipital_Inf_R, Occipital_Mid_R \\
& 943 & Precentral_L, Frontal_Inf_Oper_L, Frontal_Inf_Tri_L, Rolandic_Oper_L \\
& 936 & Putamen_L, Thalamus_L, Insula_L, Vermis_3
\end{tabular}

Note. Brain area labeling is based on the AAL atlas (> 20 overlapping voxels). 
Table 4 Results of the voxel-wise whole brain analyses on congruency effect and congruency-reading relationship

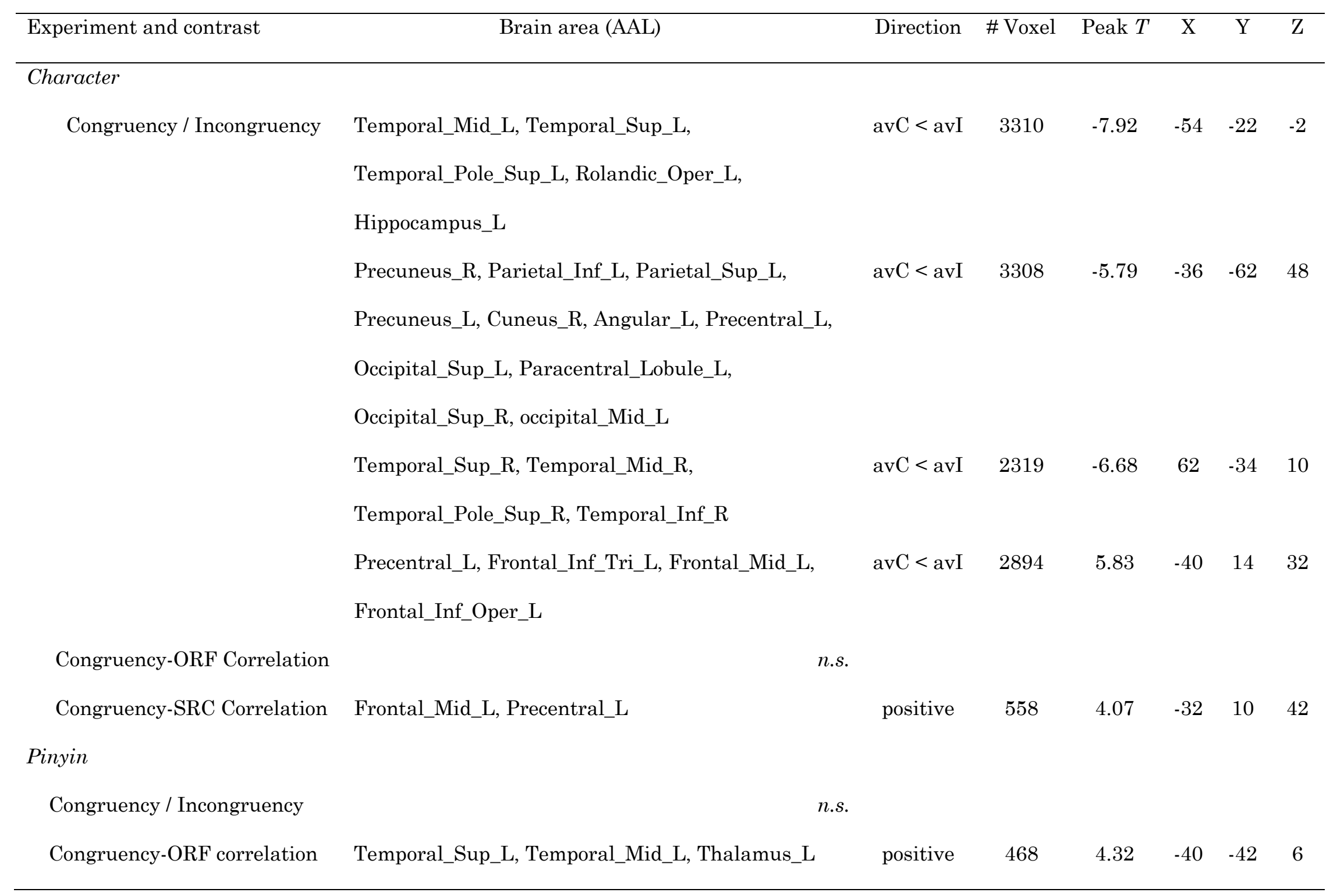


Congruency-SRC correlation n.s.

Note: Brain area labeling is based on the AAL atlas ( $>20$ overlapping voxels). Abbreviations: avC $=$ audiovisual congruent, avI $=$ audiovisual incongruent, $\mathrm{ORF}=$ oral reading fluency, $\mathrm{SRC}=$ silent reading comprehension. 


\section{A Example stimuli: character conditions}

Auditory-only
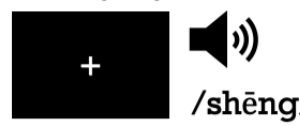

AV-congruent
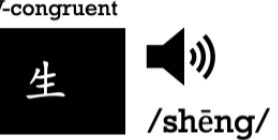

Visual-only

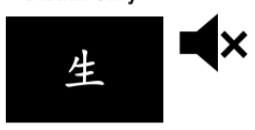

AV-incongruent

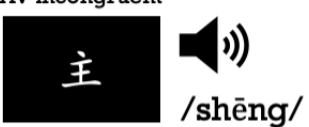

B Example stimuli: pinyin conditions

Auditory-only

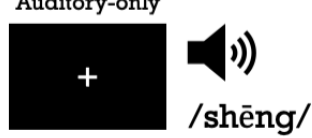

AV-congruent shēng

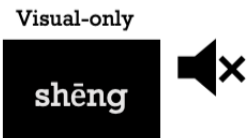

AV-incongruent

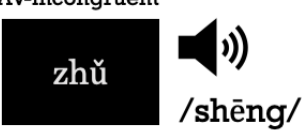

\section{C fMRI Procedure}

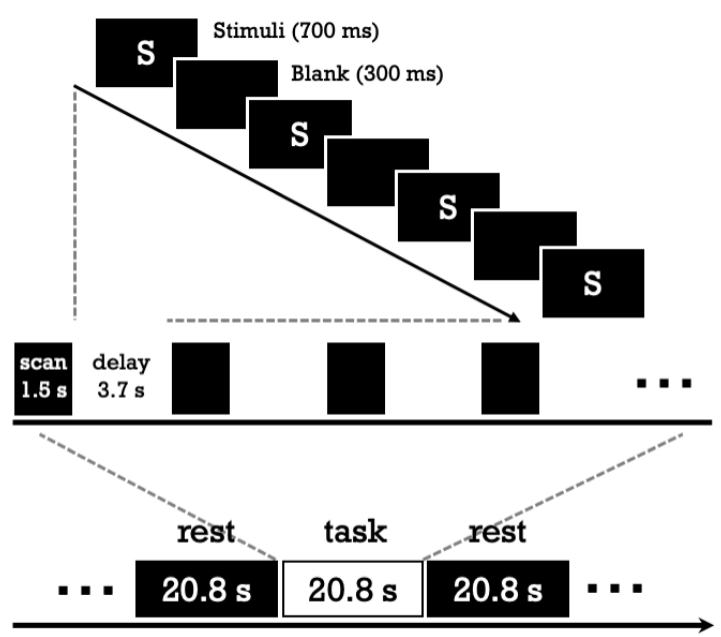

D Response targets

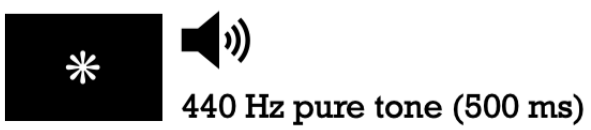

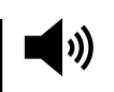

/shēng/

Figure 1 Materials and experimental design. Example stimuli used in the character

1020 (A) and pinyin (B) experiments. (C) Schematic illustration of the fMRI procedure. (D)

1021 Auditory and visual targets in the in-scanner task. 
bioRxiv preprint doi: https://doi.org/10.1101/2020.05.31.126128; this version posted June 17, 2021. The copyright holder for this preprint (which was not certified by peer review) is the author/funder, who has granted bioRxiv a license to display the preprint in perpetuity. It is made available under aCC-BY-NC-ND 4.0 International license.

Brain basis of audiovisual integration
A Additive Enhancement
Aud $>0 \cap$ Vis $>0 \cap$ avC $>($ Aud + Vis $) / 2$

\section{B Congruency Effect avC vs avI}

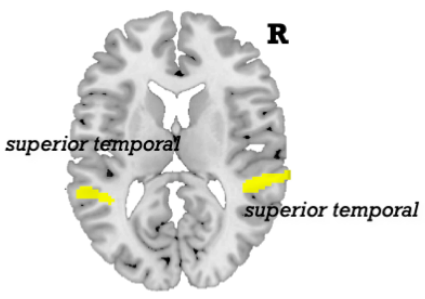

$(\mathrm{z}=12)$

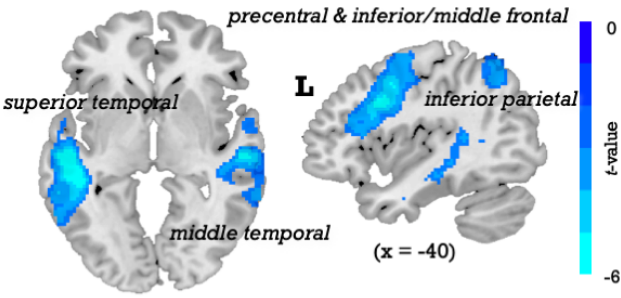

$(z=-2)$

\section{Brain-Behavior Correlation}

Brain: congruency contrast

Behavior: silent reading comprehension

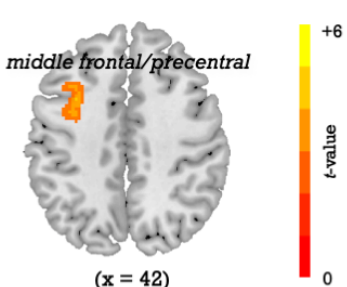

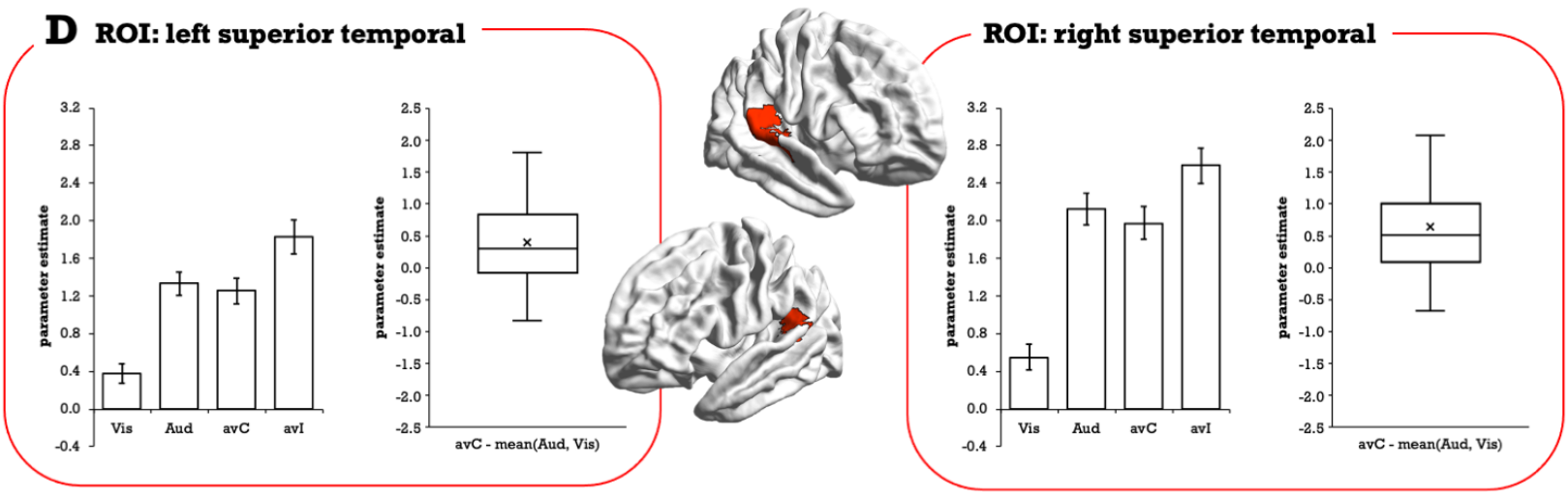

E ROI: left inferior parietal
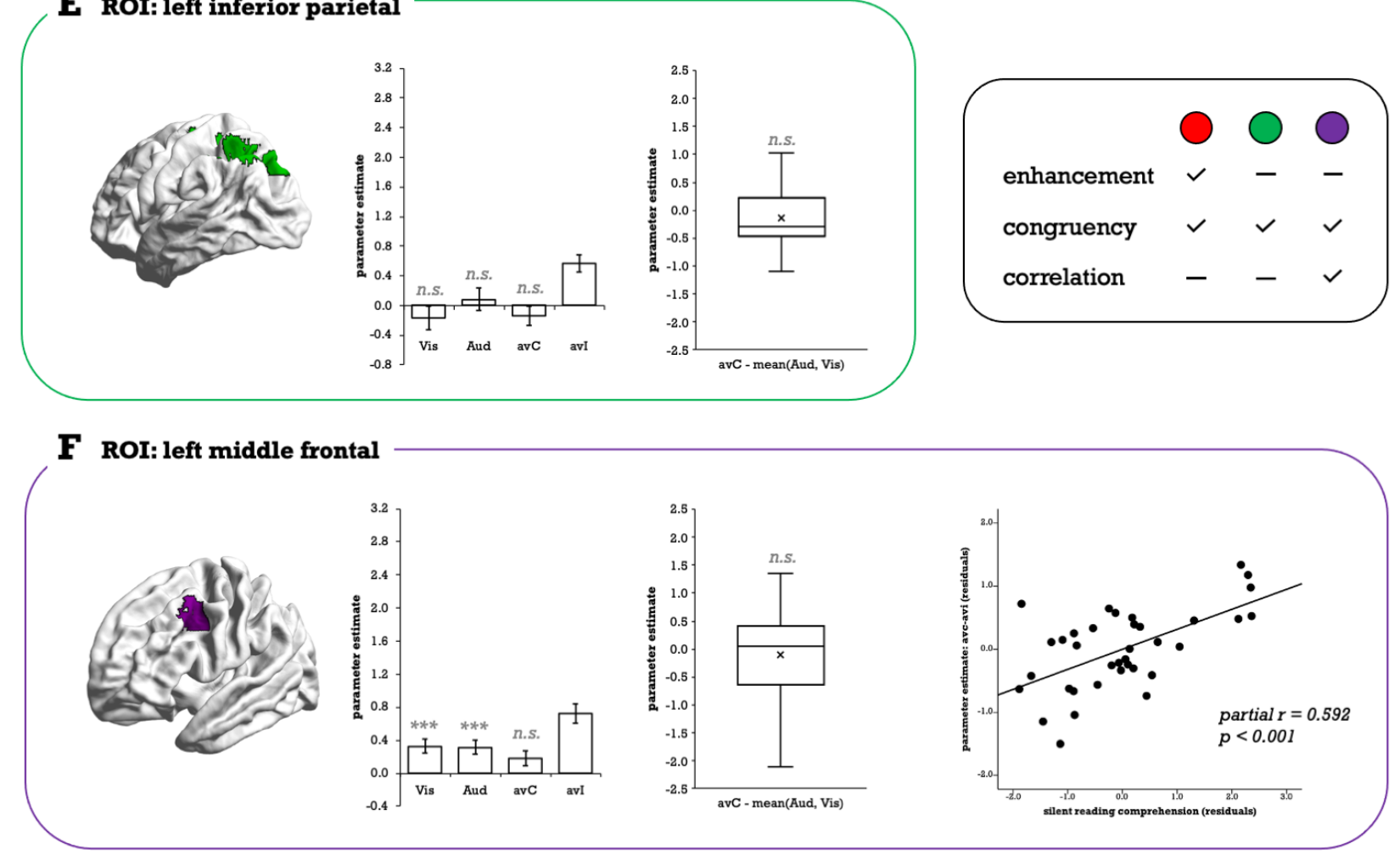

1023 Figure 2 Results of the character experiment. (A) Clusters (yellow) showing 
Brain basis of audiovisual integration

1024 audiovisual additive enhancement are identified with the mean rule (Beauchamp,

1025 2005) consisting of two criteria: (1) The region should activate in both unisensory

1026 conditions (i.e., Aud > 0, Vis > 0); (2) The audiovisual congruent stimuli should induce

1027 stronger activation than the mean activation of the unisensory conditions, i.e., avC >

1028 (Aud + Vis) / 2. An uncorrected threshold of $p$-voxel $<0.05$ is used for each contrast,

1029 resulting in a conjoint probability of $p$-voxel $<0.05^{\wedge} 3=0.000125$. A cluster containing

1030 no less than 200 continuous voxels was considered to show significant additive

1031 enhancement. (B) Areas showing a significant congruency effect (blue = incongruency,

1032 i.e., avC < avI). Threshold: cluster forming $p$-voxel < 0.005, Family-Wise Error (FWE)

1033 corrected $p$-cluster $<0.05$. (C) The region showing a significant correlation between

1034 the magnitude of congruency effect and reading scores. Threshold: cluster forming $p$ -

1035 voxel $<0.005$, FWE corrected $p$-cluster $<0.05$. (D, E, F) The areas showing both

1036 additive enhancement and congruency effect (red cluster), congruency effect only

1037 (green cluster), and both congruency effect and congruency-reading correlation (violet

1038 cluster) were defined as the regions-of-interest (ROIs). For each ROI, a bar plot

1039 displaying activation for each condition and a boxplot displaying the contrast avC -

1040 (Aud + Vis) / 2 are presented. A scatter plot is presented in the ROI showing a

1041 significant correlation between the magnitude of congruency effect and reading

1042 (residuals were obtained by regressing out nuisance variables of age and sex with a

1043 general linear model). Abbreviations: Aud = unisensory auditory, Vis = unisensory

1044 visual, avC = audiovisual congruent, avI = audiovisual incongruent, $\mathrm{L}=$ left

1045 hemisphere, $\mathrm{R}=$ right hemisphere; ${ }^{* * *} p<0.001$, n.s. $=$ non-significant. 


\section{A Additive Enhancement}

Aud $>0 \cap$ Vis $>0 \cap$ avC $>($ Aud + Vis $) / 2$

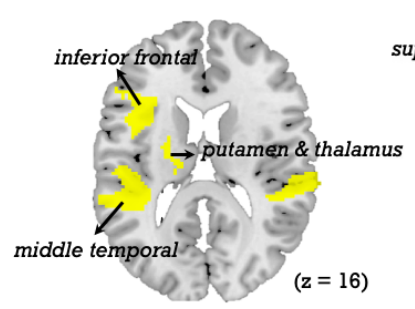

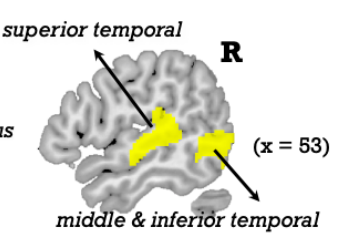

B Brain-Behavior Correlation

Brain: congruency contrast

Behavior: oral reading fluency

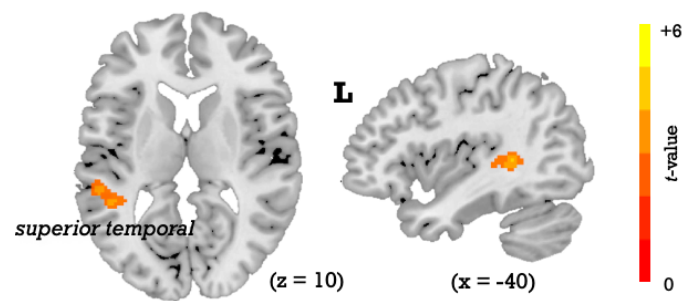

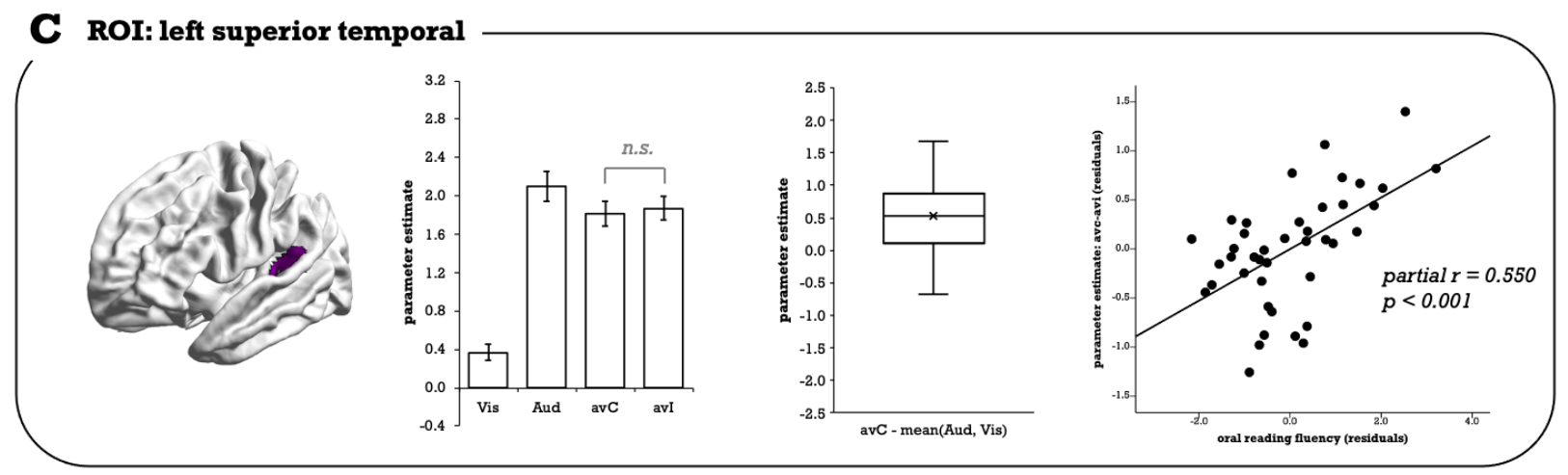

1047 Figure 3 Results of the pinyin experiment. (A) Clusters (yellow) showing audiovisual

1048 additive enhancement are identified with the mean rule (Beauchamp, 2005), which

1049 consists of two criteria: (1) The region should activate in both unisensory conditions

1050 (i.e., Aud > 0, Vis > 0); (2) The audiovisual congruent stimuli pairs should induce

1051 stronger activation than the average activation of the auditory and visual conditions,

1052 i.e., avC $>$ (Aud + Vis) / 2. An uncorrected threshold $p$-voxel $<0.05$ is used for each

1053 contrast, resulting in a conjoint probability of $p$-voxel $<0.05^{\wedge} 3=0.000125$. A cluster

1054 consisting of no less than 200 continuous voxels under the threshold is considered to

1055 show additive enhancement. (B) A cluster displaying a significant correlation

1056 between the magnitude of congruency effect and oral reading fluency. Threshold:

1057 cluster forming $p$-voxel $<0.005$, Family-Wise Error (FWE) corrected $p$-cluster $<0.05$.

1058 (C) The intersection between areas showing mean additive audiovisual enhancement 
1059 and areas showing association with reading is defined as the region-of-interest (ROI).

1060 The bar plot shows activation in each condition. There was no significant difference

1061 between the audiovisual congruent condition (avC) and incongruent condition (avI).

1062 Boxplot displays the contrast avC - (Aud, Vis) / 2. The scatter plot shows the

1063 correlation between the magnitude of congruency effect and oral reading fluency

1064 (residuals were obtained by regressing out nuisance variables of age and sex with a

1065 general linear model). Abbreviations: Aud = unisensory auditory, Vis = unisensory

1066 visual, avC $=$ audiovisual congruent, avI = audiovisual incongruent, $\mathrm{L}=$ left

1067 hemisphere, $\mathrm{R}=$ right hemisphere; n.s. = non-significant. 
$\mathbf{A}$

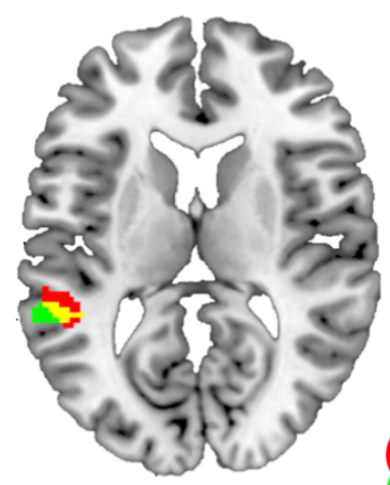

$(\mathrm{z}=12)$

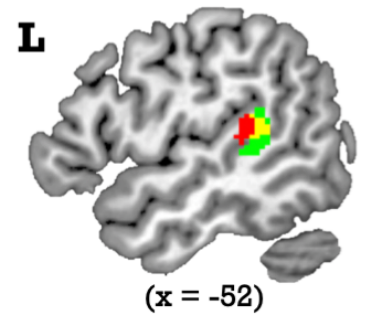

Pinyin Left STC ROI

Intersection

Character Left STC ROI
B

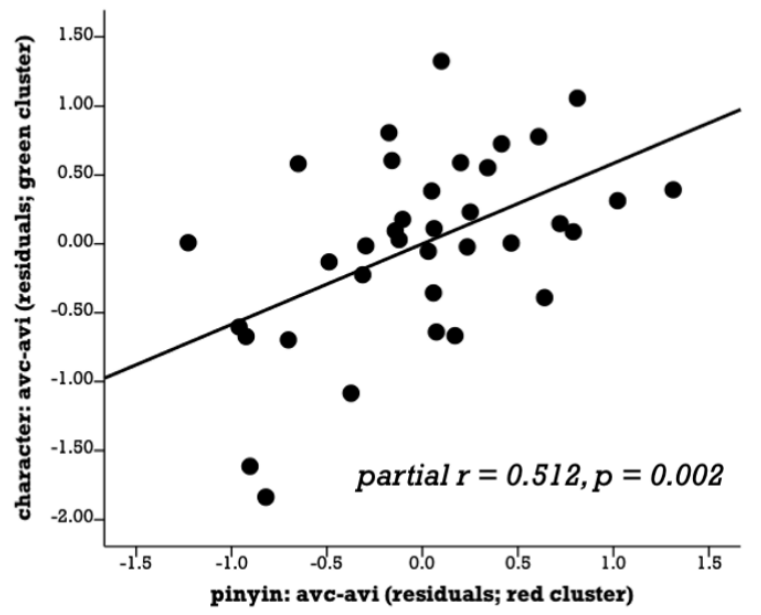

Figure 4 (A) The regions-of-interest (ROIs) in the left superior temporal cortex

1070 defined in the character experiment (green) and pinyin experiment (red), as well as

1071 their intersection (yellow), are presented on a template brain. (B) The scatter plot

1072 shows the significant correlation between the magnitude of congruency effect in the

1073 character ROI and the pinyin ROI (residuals were obtained by regressing out

1074 nuisance variables of age and sex with a general linear model). 